\title{
Geographical Distribution of Vampire Bat-related Cattle Rabies in Brazil
}

\author{
Yuki KOBAYASHI ${ }^{1}$, Ai OGAWA ${ }^{1)}$, Go SATO ${ }^{1)}$, Tetsuo SATO ${ }^{1)}$, Takuya ITOU ${ }^{1)}$, Samir I. SAMARA ${ }^{2)}$, \\ Adolorata A. B. CARVALHO ${ }^{2)}$, Darci P. NOCITI ${ }^{2)}$, Fumio H. ITO $^{3)}$ and Takeo SAKAI ${ }^{1) *}$ \\ ${ }^{1)}$ Nihon University Veterinary Research Center, 1866 Kameino, Fujisawa, Kanagawa 252-8510, Japan, ${ }^{2)}$ Faculty of Agriculture and \\ Veterinary Science, UNESP, Via de Acesso Prof. Paulo Donato Castellane, Jaboticabal, São Paulo 14884-900 and ${ }^{3)}$ Faculty of \\ Veterinary Medicine and Zootechny, University of São Paulo, Av. Prof. Dr. Orlando Marques de Paiva, 87, Cidade Universtiátria, São \\ Paulo 05508-000, Brazil
}

(Received 7 February 2006/Accepted 1 June 2006)

ABSTRACT. Seventy-seven rabies virus (RV) isolates originating from Brazilian cattle were genetically characterized. Partial nucleoprotein gene sequences of these isolates were phylogenetically and geographically analyzed. Cattle isolates, which clustered with the vampire bat-related RV group, were further subdivided into nine genetic subgroups. These subgroups were distributed widely in lowland regions, with some subgroups separated from each other by mountain ranges. In addition, separation of the groups in mountainous regions was correlated with altitude. These results indicate that cattle rabies is derived from several regionally-defined variants, which suggests that its geographical distribution is related to that of the vampire bat population.

KEY WORDS: cattle, geographical analysis, rabies.

J. Vet.Med. Sci. 68(10): 1097-1100, 2006

The rabies virus (RV), which belongs to genotype 1 of the Lyssavirus genus within the Rhabdoviridae family, has an almost global distribution throughout the world. The principal RV reservoirs are known to differ between regions in the areas where the virus occurs, and dogs and vampire bats (Desmodus rotundus) are the major known RV vectors in Brazil. Vampire bats, which are widely distributed in Latin America, transmit RV to herbivore species and are recognized as an important RV vector in herbivore rabies [2, 3, 10]. Vampire bats live in small colonies of 10 to 300 animals and range within an area of activity of between 10 and 20 $\mathrm{km}^{2}[2,14]$. During the period of 1993-2002, 31,187 cases of cattle rabies were reported in Latin America, and vampire bat-transmitted RVs have had an economic impact in the livestock industry $[4,10]$. In this study, to define the epidemiological characteristics of vampire bat-transmitted rabies, we performed a phylogenetic analysis and related the findings to the geographical distribution of 86 vampire bat-transmitted RV isolates from cattle in several states in Brazil.

Seventy-seven of the RV isolates were collected from cattle in the city of Brasilia, the Federal District, and the states of Goiás, Maranhão, Minas Gerais, Mato Grosso, Rondônia, Pará, São Paulo, and Tocantins between 1998 and 2003 (Table 1). Additionally, nine isolates were collected from cattle (BRbv30, 32, 36, 38, 39, 43, 45, 49, and $50)$, eight isolates were collected from vampire bats (BRvmbt33, 34, 41, 46 and 47, and BR-DR1-3), and two isolates were collected from $\operatorname{dogs}$ (BRdg10 and 12) as described previously [7, 8]. All cattle isolates were identified as vampire bat-related RV by sequencing and phylogenetic analysis. Viral RNA was extracted from the brains of cattle diagnosed as RV positive by both the direct fluores-

\footnotetext{
* Correspondence to: Prof. SaKai, T., Nihon University Veterinary Research Center, 1866 Kameino, Fujisawa, Kanagawa 2528510 , Japan.
}

cence antibody test [5] and the mouse inoculation test [9]. RT-PCR was used to amplify nucleoprotein $(\mathrm{N})$ gene sequences of the Brazilian RV strain as described previously [8] using P1/P2 primer pairs. Sequencing was performed using the methods described in our previous study [8]. The 203 nucleotide sequences corresponding to positions 109 311 of the $\mathrm{N}$ gene sequence of the PV strain were determined using the $\mathrm{P} 1$ and BRABN-C3 primers. This locus has been reported to be associated with the phylogenetic divergence of clusters and has been used in other phylogenetic studies $[1,7]$. The nucleotide sequences were aligned using the Clustal X program [13], and a neighbor-joining tree was constructed using the method of Saitou and Nei [12]. Bootstrap values were calculated using 1,000 replicates, and homologies between nucleotide sequences were identified using the BioEdit software [6].

The nucleotide sequences of the 203 RT-PCR products corresponding to the RV N gene were determined for all 77 $\mathrm{RV}$ isolates and were deposited in GenBank (Accession numbers AB246194-246270). The cattle isolates were clustered in nine major subgroups characterized by high nucleotide identities (above 93\%) except for a small number of the cattle isolates and these subgroups were distributed in limited areas associated with geomorphology (Fig. 1). The geographic locations of the isolates belonging to the nine subgroups are shown in Fig. 2. Subgroup A consisted of 9 samples from the lowland regions of northern Tocantins and its neighboring states. These samples were distributed among different hydrographic basins separated by several rivers. Subgroup B consisted of three samples from lowlying mountainous regions approximately $300 \mathrm{~km}$ to the south of subgroup A. Subgroup C consisted of 13 samples from Brasília and eastern Goiás. These isolates were distributed along low altitude areas in mountainous ranges. Subgroup D consisted of eight cattle and three vampire bat 
Table 1. List of Brazilian cattle isolates used for the phylogenetic analysis

\begin{tabular}{ll}
\hline Place of isolation & Identification number \\
\hline Brasília city & BRbv296 \\
Goiás state & BRbv30 $^{\text {a) }}, 50^{\text {a) }}, 56,80,82,86,89,93,94,95,103,106,119$, \\
& $124,132,138,141,145,147,150,152,153,157,160,161$, \\
& $162,169,170,181,182,185,187$ \\
Maranhão state & BRbv55 \\
Mato Grosso state & BRbv43 ${ }^{\text {a) }}, 45^{\text {a) }}, 57,186,192,193,194,195,197,200,202$, \\
& $206,207,208,210,214,216,217,221,225,229,230,232$, \\
Minas Gerais state & $234,235,238$ \\
Rondônia state & BRbv63,74,282,287 \\
Pará state & BRbv76 \\
São Paulo state & BRbv32 ${ }^{\text {a) }}, 49^{\text {a) }}, 62,64,65,66,69,71,75,118$, \\
Tocantins state & BRbv36 ${ }^{\text {a) }}, 38^{\text {a) }}, 39^{\text {a) }}, 87,129,133,140,168,178,183$, \\
\hline
\end{tabular}

a) The following data were obtained from GenBank: BRbv30 (AB083803), BRbv32 (AB083805), BRbv36 (AB083809), BRbv38 (AB083810), BRbv39 (AB083811), BRbv43 (AB083813), BRbv45 (AB083814), BRbv49 (AB083817), and BRbv50 (AB083818)

isolates from mountainous regions at altitudes below $800 \mathrm{~m}$ in the states of Goiás and São Paulo, but the strength of the node in the phylogenetic tree was low (bootstrap values of 307). Subgroup E consisted of 18 samples that were distributed over an extensive area that included both the mountainous and lowland regions of the states of Goiás and Mato Grosso. Subgroup F consisted of nine samples from the mountainous regions of central Goiás. These isolates were distributed in low altitude regions and were separated from the isolates in subgroup $\mathrm{C}$ and $\mathrm{E}$ by high mountains. Subgroup $\mathrm{G}$ consisted of four samples from lowland regions surrounding mountainous areas between the state of Goiás and Mato Grosso from which subgroups E and F were isolated. Subgroup H consisted of isolates from six cattle and two vampire bats from mountainous regions between the state of Minas Gerais and São Paulo. These samples were isolated in areas that were higher in altitude compared to subgroup D isolated in São Paulo state. Interestingly, although subgroup I consisted of 8 widely distributed isolates from a large lowland region between the state of Goiás and Mato Grosso, the nucleotide identities were $100 \%$.

Phylogenetic analysis of the $\mathrm{N}$ gene showed that the cattle isolates were genetically related to the vampire bat RV group and that they formed a clade consisting of nine subgroups. The geographical distributions of each subgroup were separated by mountains, with additional differences observed between the lowland and mountainous regions. The separation attributed to mountain ranges was particularly apparent in subgroups C, E, and F. Although the samples of these subgroups were isolated in areas neighboring each other, the samples could be divided into three phylogenetic groups by the distribution of mountains in the regions where the samples were isolated. In the lowland regions, cattle isolates were widely distributed and separated from the other subgroups by mountains. Epidemiological evidence of herbivore rabies suggests that the clusters originated from the same hydrographic basin in the state of Rio de Janeiro [11]. Furthermore, it has been suggested that bats need to fly longer distances in order to feed in large regions, and that spread of the virus may thus occur in a shorter period of time [11]. In this study, the flatland regions from which subgroups A, E, and I were isolated are characterized as having numerous rivers. However these groups were not separated by rivers and had widespread distributions. These findings, corroborated by Romijn et al. [11], suggest that vampire bat-related RV tends to spread in lowland regions. The formation of each subgroup observed in the mountainous regions was correlated with altitude. Subgroup H, which was distributed between 800 and 1,600 m, was separated from subgroup D, which was located at lower altitudes. Furthermore, two vampire-bat isolates, BR-DR1 and 2 , were distributed in this area. Vampire bats are known to inhabit areas at altitudes below 2,000 m [3]. In high altitude areas, vampire bat populations may retain specific RV variants.

The present study indicates that cattle rabies is derived from several variants that are regionally well-defined. This finding implies that the geographical distribution of cattle rabies is dependent on that of the vampire bat populations because the major transmitter of cattle rabies is vampire bats [3] and vampire bats live in colonies with a limited migratory range $[2,14]$. Moreover, the transmission of vampire bat-related RV appears more prevalent in flatland regions and is limited by the distribution of mountains. Similarly, RV circulation was observed to be related to altitude. This therefore reveals that the geographic isolation of bat populations, especially in mountainous and lowland areas, is an important factor in the development of the epidemiological characteristics of vampire bat-transmitted RV.

ACKNOWLEDGMENTS. This work was partly supported by the Academic Frontier Project for Private Universities from the Ministry of Education, Culture, Sports, Science and Technology (MEXT) of Japan, the Grant-in Aid for Sci- 


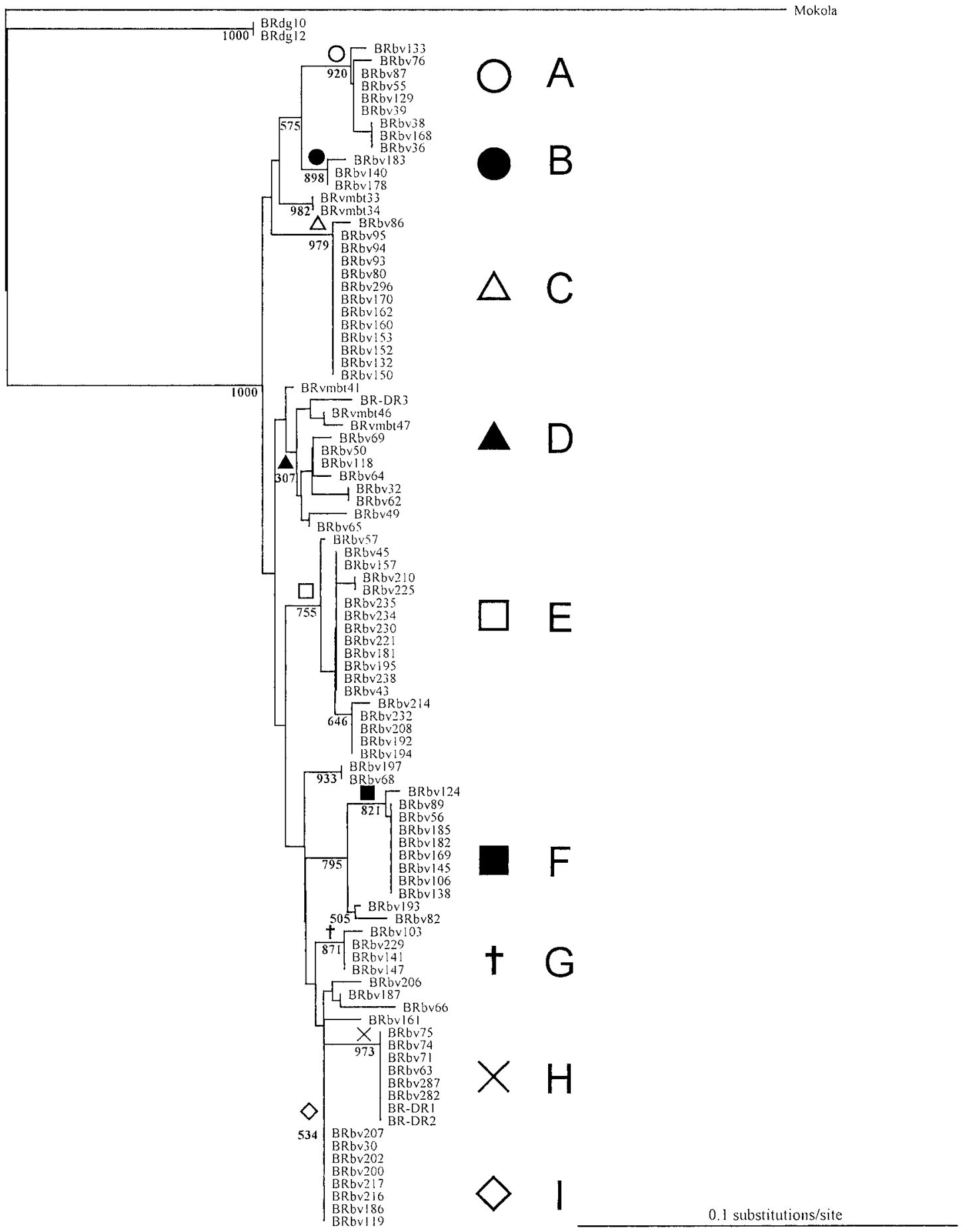

Fig. 1. Phylogenetic tree based on a partial N gene sequences. Mokola virus was used as outgroup. The Brazilian dog isolates are as follows: BRdg10 (AB083796) and BRdg12 (AB083797). The vampire bat isolates are as follows: BRvmbt33 (AB083806), BRvmbt34 (AB083807), BRvmbt41 (AB083812), BRvmbt46 (AB083815), BRvmbt47 (AB083816), BRDR1 (AB201803), BR-DR2 (AB201804), and BR-DR3 (AB201805). These isolates were employed in previous reports (Ito et al. 2001 and Kobayashi et al. 2005). The symbols correspond to the nine subgroups (groups A to I). Bootstrap values, determined from 1,000 replicates of the data, are shown to the left of all major branches. 


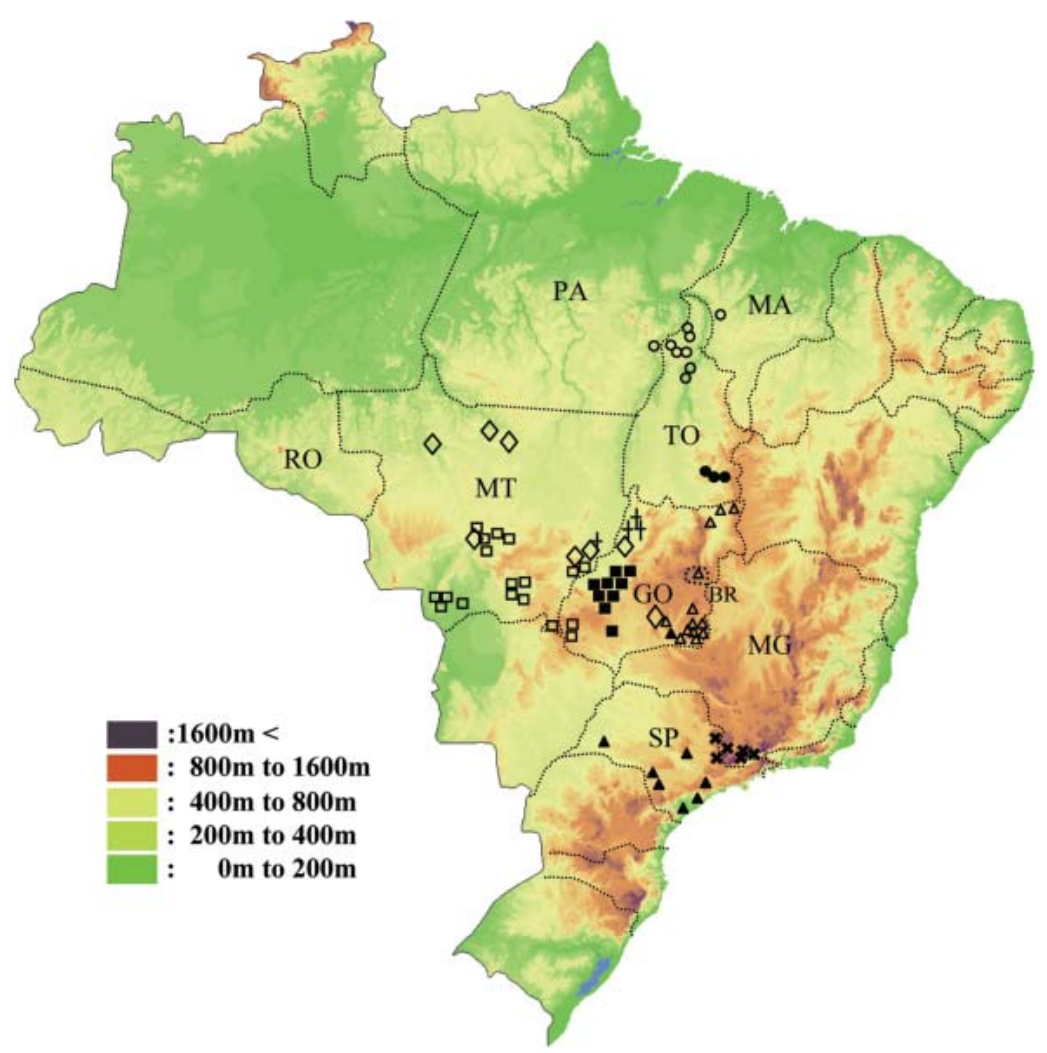

Fig. 2. Geographical distribution of cattle isolates clustered into nine subgroups in the phylogenetic analysis. The symbols correspond to Fig. 1. Subgroup A, $\bigcirc$; subgroup B, $\bigcirc$; subgroup C, $\triangle$; subgroup D, $\mathbf{\Delta}$; subgroup E, $\square$; subgroup F, $\square$; subgroup G, +; subgroup H, $\times$; subgroup I, $\diamond$. City and state abbreviations are as follows: BR, Brasília city; GO, Goiás State; MA, Maranhão State; MG, Minas Gerais State; MT, Mato Grosso State; RO, Rondônia State; PA, Pará State; SP, São Paulo State; TO, Tocantins State.

entific Research B from Japan Society for the promotion Science, and the grant for the Research on Emerging and Re-emerging Infectious Diseases, Ministry of Health, Labour and Welfare, Japan.

\section{REFERENCES}

1. Arai, Y.T., Yamada, K., Kameoka, Y., Horimoto, T., Yamamoto, K., Yabe, S., Nakayama, M. and Tashiro, M. 1997. Arch. Virol. 142: 1787-1796.

2. Arellano-Sota, C. 1988. Rev. Infect. Dis. 10 (Suppl. 4): S615619.

3. Arellano-Sota, C. 1988. Rev. Infect. Dis. 10 (Suppl. 4): S707709.

4. Belotto, A., Leanes, L.F., Schneider, M.C., Tamayo, H. and Correa, E. 2005. Virus Res. 111: 5-12.

5. Dean, D. J., Ableseth, M. K. and Atanasiu, P. 1996. pp. 88-95. In: Laboratory Techniques in Rabies, 4th ed. (Meslin, F. -X., Kaplan, M. M. and Koprowski, H. eds). World Health Organi- zation Geneva.

6. Hall, T.A. 1999. Nucleic Acids Symp. Ser. 41: 95-98.

7. Ito, M., Arai, Y.T., Itou, T., Sakai, T., Ito, F.H., Takasaki, T. and Kurane, I. 2001. Virology 284: 214-222.

8. Kobayashi, Y., Sato, G., Shoji, Y., Sato, T., Itou, T., Cunha, E.M., Samara, S.I., Carvalho, A.A., Nociti, D.P., Ito, F.H. and Sakai, T. 2005. J. Vet. Med. Sci. 67: 647-652.

9. Koprowski, H. 1996. pp. 80-87. In: Laboratory Techniques in Rabies, 4th ed. (Meslin, F. -X., Kaplan, M. M., and Koprowski, H. eds), World Health Organization Geneva.

10. Mayen, F. 2003. J. Vet. Med. B Infect. Dis. Vet. Public Health 50: 469-472.

11. Romijn, P.C., van der Heide, R., Cattaneo, C.A., Silva Rde, C. and van der Poel, W.H. 2003. Am. J. Trop. Med. Hyg. 69: 8186.

12. Saitou, N. and Nei, M., 1987. Mol. Biol. Evol. 4: 406-425.

13. Thompson, J.D., Gibson, T.J., Plewniak, F., Jeanmougin, F. and Higgins, D.G. 1997. Nucleic Acids Res. 25: 4876-4882.

14. Wimsatt, W.A. 1969. J. Mamml. 50: 233-244. 